\title{
Lack of change in glucose metabolism in eszopiclone-treated primary insomnia patients
}

\author{
Orfeu M Buxton ${ }^{1-4}$ \\ Milena K Pavlova ${ }^{1,5}$ \\ Shawn P O'Connor' \\ Wei Wang ${ }^{1,2}$ \\ John W Winkelman ${ }^{1,6}$
}

'Division of Sleep Medicine, Harvard Medical School, ${ }^{2}$ Department of Medicine, Brigham and Women's Hospital, ${ }^{3}$ Department of Social and Behavioral Sciences, Harvard School of Public Health, Boston, MA, ${ }^{4}$ Department of Biobehavioral Health, Pennsylvania State University, University Park, PA, ${ }^{5}$ Department of Neurology, Brigham and Women's Hospital, 'Department of Psychiatry, Massachusetts General Hospital, Boston, MA, USA
Correspondence: Orfeu M Buxton Department of Biobehavioral Health, Pennsylvania State University, 22I

Biobehavioral Health BIdg, University

Park, PA 16802, USA

Tel+I $8 \mid 48675707$

Fax+l 6175079177

Email Orfeu@PSU.edu
This article was published in the following Dove Press journal:

Nature and Science of Sleep

18 July 2017

Number of times this article has been viewed

Study objectives: Primary insomnia (PI) may increase diabetes risk. We tested the hypothesis that the effects of PI on glucose metabolism could be improved by 2 months of pharmacological treatment.

Methods: Adult men and women meeting clinical criteria for PI were studied ( $n=20$, body mass index $25.1 \pm 2.7 \mathrm{~kg} / \mathrm{m}^{2}$, age $39.7 \pm 7.9$ ) in a randomized, double-blind, placebo-controlled clinical trial. The study consisted of two 1-day inpatient admissions to a General Clinical Research Center separated by 2 months of at-home treatment with $3 \mathrm{mg}$ eszopiclone or placebo. During inpatient admissions, each subject underwent two intravenous glucose tolerance tests (IVGTTs) pre- and post-treatment. Diet was controlled for micro- and macro-nutrient content and calories on the day prior to pre- and post-treatment IVGTTs. Subjects were randomized following completion of the initial IVGTT to take either placebo or eszopiclone $30 \mathrm{~min}$ prior to bedtime at home for $2 \mathrm{months}$. Results: Two-month eszopiclone treatment did not change insulin sensitivity, glucose tolerance, or any of the sleep measures significantly, compared with placebo. Changes in glycated hemoglobin (HbA1c, clinical measure of glycemic control) were correlated with changes in diary-reported total sleep time in the eszopiclone group $(r=0.66, p=0.0360)$, and in the combined groups' data $(r=0.55, p=0.0125)$. Changes in polysomnography-measured wake after sleep onset, a hallmark of PI, were positively related to changes in IVGTT-derived glucose effectiveness, or non-insulin-mediated glucose uptake.

Conclusion: Treatment with $3 \mathrm{mg}$ eszopiclone for 2 months, compared with placebo, did not significantly influence either sleep or measures of diabetes risk in this preliminary study.

Keywords: primary insomnia, sleep duration, metabolism, IVGTT, insulin sensitivity, diabetes, eszopiclone, wake after sleep onset

\section{Introduction}

Reduced sleep time independent of insomnia diagnosis has been associated with a variety of deleterious long-term effects, including an increased risk of higher weight ${ }^{1}$ and symptomatic diabetes. ${ }^{2}$ In meta-analysis of sleep and diabetes risk, insomnia symptoms were even more strongly related to diabetes than short sleep duration; difficulty initiating or maintaining sleep was associated with an elevated relative risk of 1.57 and 1.84 , respectively. ${ }^{3}$ A more recent meta-analysis systematically evaluating a variety of sleep disturbances identified poor sleep quality as an independent predictor of type 2 diabetes, and recommended including sleep disturbances in screening for type 2 diabetes. ${ }^{4}$ Despite many consequences of insomnia for health and productivity, sleep disorders are not commonly evaluated in primary care. ${ }^{5}$ 
Current understanding of the pathophysiology of insomnia includes the "hyperarousal" hypothesis, positing that the difficulties with sleep initiation and/or maintenance arise from a combination of biological and psychological traits and cognitive attitudes toward sleep. The classical "3 $\mathrm{P}$ " model $^{7}$ postulates that chronic insomnia develops in individuals with a predisposition (due to inborn or other factors), that may be activated from a precipitating factor (medical illness or psychological stress) and be self-reinforced by perpetuating factors (maladaptive behaviors, anxiety related to sleep and dysfunctional belief about sleep). The end result is a state of heightened arousal throughout the day that continues into the sleep hours. The state of hyperarousal from molecular to higher system levels is accepted as a model of insomnia. ${ }^{7}$ A heightened state of arousal, often expressed as rumination, inability to stop thinking about day's events at night, or a general sense of continuous alertness ("I simply cannot switch off, doctor") is often subjectively reported by insomnia patients, ${ }^{7,8}$ and may vary with gender. ${ }^{9}$ This condition has been reported to affect objectively-assessed sensory processing $^{11}$ and correlate with various spectral electroencephalographic measures. ${ }^{11}$

During non-rapid eye movement sleep, patients with insomnia have persistent activity of wake-promoting structures, ${ }^{12}$ and a reduction in central gamma-aminobutyric acid neurotransmission. ${ }^{1}$ Thus, it could be expected that the effects of this heightened state of arousal could also trigger an activation of the hypothalamic-pituitary-adrenal axis in a way similar to severe physiologic or psychological stress, ${ }^{13}$ with a consequent increase of serum glucose and a potential for impaired glucose tolerance.

The reversibility of insomnia-associated impairments of glucose metabolism is unknown. In this study, we assessed primary insomnia (PI) patients for their baseline HbA1c levels and responses to an intravenous glucose tolerance test (IVGTT) to yield measures of glucose tolerance relevant for clinical diagnoses of diabetes type $2(\mathrm{DM} 2)^{14}$ and research evaluations of impaired glucose tolerance, ${ }^{15}$ pancreatic beta cell secretion of insulin in response to a glucose load, and insulin sensitivity $\left(\mathrm{S}_{\mathrm{I}}\right)$, the response of peripheral tissues to insulin in storing glucose. We then tested the hypothesis that chronic PI is associated with impairments of glucose metabolism that can be reversed by 2 months of treatment with eszopiclone for the PI. Finally, we test the hypothesis that, in patients with PI, changes in actigraphic or polysomnographic or polysomnography (PSG)-measured sleep are related to changes in glucose metabolism related to the anticipated improvements in sleep with an anti-insomnia medication.

\section{Methods}

Institution where the study was performed: Brigham and Women's Hospital, Boston, MA, USA.

\section{Study design}

A schematic of this double-blind, placebo-controlled, randomized clinical study is presented in Figure 1. The procedures were approved by the Human Research Committee of the Brigham and Women's Hospital and conducted according to the principles expressed in the Declaration of Helsinki. All subjects provided written informed consent.

\section{Subject recruitment and screening}

Young and middle-aged (25-55 years) individuals with PI were recruited through advertisements for a study of glucose metabolism and neuroimaging ${ }^{1}$ in Diagnostic and Statistical Manual of Mental Disorders, 4th Edition (DSM-IV) defined PI (307.42) at Brigham and Women's Hospital from May 2006 to May 2008. Subjects were required to have $>6$ months of difficulty initiating or maintaining sleep, with resulting daytime distress or dysfunction. Specifically, subjects reported at screening a total sleep time (TST) $\leq 6.5 \mathrm{~h}$, and a) sleep onset latency (SOL) $>45 \mathrm{~min}$, or b) wake after sleep onset (WASO) $>45 \mathrm{~min}$, or c) total wake time during the sleep period (sleep latency + WASO) $>60 \mathrm{~min}$.

A structured clinical assessment was performed by a single investigator (JWW), providing history of medical illnesses, other comorbid sleep disorders, as well as interview for current and lifetime history of psychiatric disorders with the Structured Clinical Interview for DSM-IV (SCID). Upon starting the study, all subjects underwent physical examination by a licensed physician, and provided blood and urine samples to ensure that hematology and serum chemistry, including metabolic and thyroid panels, were within normal limits. All subjects passed a urine toxicology screen.

Additional evaluations in all subjects included assessment with an unstructured clinical interview for history of medical and sleep disorders, and interview for lifetime history of psychiatric disorders with the SCID. A full in-laboratory (PSG) was performed to screen for comorbid primary sleep disorders other than PI. The Pittsburgh Sleep Quality Inventory (PSQI), the Beck Depression Inventory, and Insomnia Severity Index (ISI) were self-administered by all subjects. Laboratory assessment included electrolytes, complete blood count, liver and thyroid functions, pregnancy testing, and a toxicology screen for illicit substances.

\section{Pre-study conditions}

Sleep diaries were completed by all subjects, supplemented by daily call-ins at bedtime and wake time, to assess the 
3-week, at-home wrist actigraphy

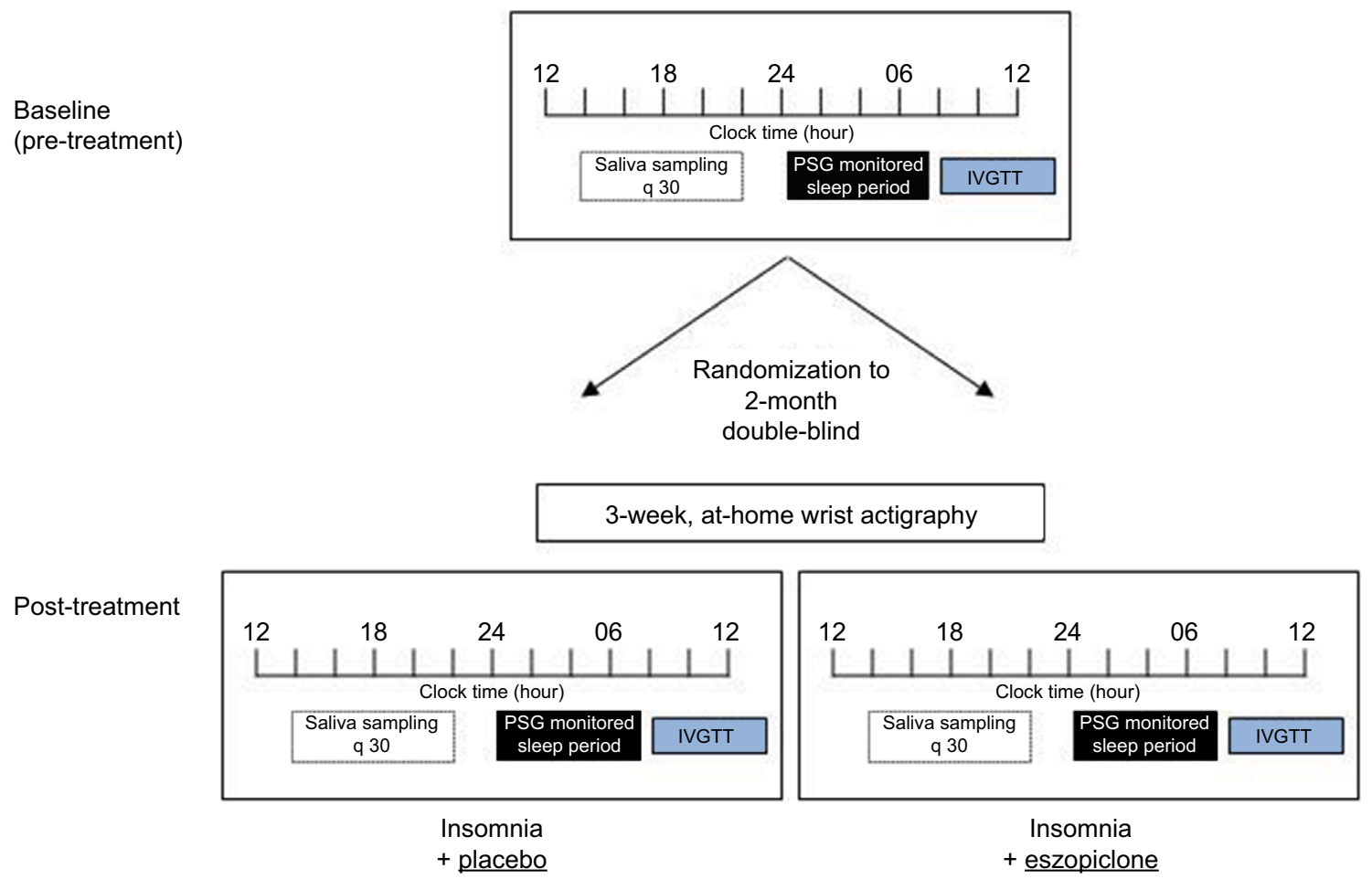

Figure I Protocol schema.

Abbreviation: IVGTT, intravenous glucose tolerance test; PSG, polysomnography; q 30, every 30 minutes.

timing of sleep and wake onset, TST and awakenings within the sleep episode in all subjects. Inclusion criteria described above for SOL, WASO and TST had to be met during the screening period, prior to the clinical assessment.

Exclusion criteria included recent (within the preceding year) or current diagnosis of a DSM-IV Axis I disorder (including drug or alcohol abuse) besides PI; symptoms, diagnosis, or history of any sleep disorder other than PI; history of significant head trauma (e.g., loss of consciousness $>30 \mathrm{~min}$ ); body mass index (BMI) $>32$ or $<19.8 \mathrm{~kg} / \mathrm{m}^{2}$; regular treatment (more than once per week) with CNS-active medications within 3 months of the first visit; current smoking of $>10$ cigarettes/day, consumption of $>2$ caffeinated beverages per day; $>2$ alcoholic drinks per day (for $>1$ month) within the preceding year; and work history of swing shift, night shift, or rotating shift within the preceding year.

\section{Actigraphy}

Each subject was made to wear an actigraphy monitor (Actiwatch AW-64; Minimitter Inc., Bend, OR, USA) on the non-dominant wrist for the duration of the study. During the screening phase of this study, these data were primarily used to verify sleep-wake diary information and not for independent assessment of inclusion and exclusion criteria. Actiware software version 3.400 .10 was used to program the Actiwatch to record activity and to download data from the device. Analysis was performed on the data collected in the 3 weeks prior to each inpatient visit using the manufacturer's algorithm in Actiware software version 5.57.0006 as validated versus PSG. ${ }^{16}$ The variables assessed for this study included time in bed (TIB), TST, SOL, WASO and sleep efficiency (SE). Analysis intervals were determined using bedtimes and wake times reported in daily diaries. Daily callins to a time-stamped voice mailbox were also used to assist with determination of bed times and wake times when diary information was absent. The threshold to detect wakefulness within the sleep period was set to low sensitivity. Sleep onset was determined to be the first epoch of the first sequence of ten consecutive epochs scored as sleep within the designated analysis interval. Sleep end was marked as the final epoch of the last sequence of five consecutive epochs scored as sleep within that same interval.

\section{Polysomnography}

Subjects who met initial screening criteria for insomnia underwent one night of attended in-laboratory screening PSG to rule 
out primary sleep disorders (sleep screen) and two additional nights for assessment of sleep architecture (inpatient PSG 1 and PSG 2). Inpatient PSG 1 and PSG 2 were performed 62.7 days (range 54-84) apart on an average. Sleep screen PSGs were conducted using either Vitaport-3 (TEMEC Instruments B.V., Kerkrade, the Netherlands) or Alice IV (Respironics, Murrysville, PA, USA) digital sleep recorders. Inpatient PSGs used Vitaport-3 digital sleep recorders only. Surface electrodes (Beckman Instrument Company, Schiller Park, IL, USA) were applied for recording central (C3 and $\mathrm{C} 4)$ and occipital (O1 and $\mathrm{O} 2$ ) electroencephalogram, electrooculogram, anterior tibialis and submentalis electromyogram, and electrocardiogram. Respiratory measures were conducted via oximetry and respiratory effort (abdominal and thorax), flow and nasal pressure. Anterior tibialis and respiratory recordings were only performed during the sleep screen. Lights out occurred at the subjects' usual time and all subjects were studied for $8 \mathrm{~h}$. In the following paragraphs, it is explained that we used the midpoint of the subjects' sleep periods to determine timing. All sleep recordings were scored according to current American Academy of Sleep Medicine criteria by the same, experienced registered polysomnographic technologist. More than 15 apnea + hypopneas or 20 periodic limb movements per hour of sleep led to exclusion from the study. Similarly, SE $>90 \%$ on the sleep screening PSG combined with a report of sleep similar to that at home was exclusionary, as potential evidence of paradoxical insomnia. Other than these exclusionary criteria, the results of PSG were not used to confirm a diagnosis of PI.

\section{Inpatient study conditions}

Subjects were admitted to the General Clinical Research Center (GCRC) at Brigham and Women's Hospital for a 1-day pre-treatment inpatient visit. Sleep periods were scheduled for $8 \mathrm{~h}$, centered at the midpoint of each subject's habitual sleep period. Light levels during sleep periods were essentially complete darkness ( $<1$ lux) and $<90$ lux during wakefulness, which simulations suggest would lead to a $<9$ min mean difference of circadian phase between sleep conditions. ${ }^{17}$ Metabolic assessments were performed, and subjects were discharged in the afternoon of day 2 (Figure 1). Metabolic assessments are described below and consisted of an intravenous glucose tolerance test (IVGTT; insulin-modified), and collection of saliva and urine for hormone measurements.

\section{Treatment and randomization}

Following successful completion of the baseline procedures, subjects were randomized by the Investigational Drug Service of the Brigham and Women's Hospital to eszopiclone (3 mg) tablets or placebo tablets, with ten subjects being randomized to eszopiclone. Each subject was instructed to maintain habitual sleeping habits but to take the tablets at home 30 min prior to bedtime for 2 months between visits without gaps. Because of scheduling logistics, visits ranged from 54 to 79 days apart (mean \pm standard deviation [SD] of $61.3 \pm 6.7$ days). Pill counts were performed at the end of treatment to confirm treatment adherence in all subjects. Subjects then returned to the GCRC for the post-treatment visit, repeating the same procedures as baseline.

\section{Diet}

Throughout the inpatient portions of the study, subjects received an isocaloric, controlled-nutrient diet containing 58\%-60\% carbohydrates, $15 \%-17 \%$ protein, $25 \%-27 \%$ fat $( \pm 1 \%), 800-1000$ $\mathrm{mg}$ calcium, $100 \mathrm{mEq}( \pm 2 \mathrm{mEq})$ potassium, and $200 \mathrm{mEq}( \pm 2$ $\mathrm{mEq}$ ) sodium. Subjects were required to consume all the food provided. On the mornings of the inpatient visits, subjects were asked to consume breakfast at home from a menu that was included in the admission day's calculated diet. An identical menu was provided for both inpatient visits. Diet was not strictly controlled during at-home treatment; however, subjects were instructed not to significantly alter their typical diet.

Intravenous glucose tolerance test (insulin-modified) IVGTT studies were performed after an overnight fast immediately following the sleep period during each inpatient visit, as previously described. ${ }^{18}$ Blood samples were drawn via an intravenous catheter every $5 \mathrm{~min}$ for $20 \mathrm{~min}$ starting at $\mathrm{T}=-20$ $\mathrm{min}$. At time $0,0.3 \mathrm{~g} / \mathrm{kg}$ glucose was administered over $1 \mathrm{~min}$ as a bolus via an intravenous catheter in the non-sampling arm. Blood samples were then taken at 2, 3, 4, 5, 6, 8, 10, $12,14,16,19,21,22,24,26,28,30,35,40,45,50,55,60$, $70,80,90,100,120,140,160$, and $180 \mathrm{~min}$. At time $20 \mathrm{~min}$, Novolin R insulin $(0.02 \mathrm{U} / \mathrm{kg})$ was administered intravenously over 1 min. Minimal Model analyses (Minmod Millennium 2000, R. Bergman, University of Southern California, Los Angeles, CA) were performed to determine the acute insulin response to glucose (AIRg; first phase area under the insulin curve from $0 \mathrm{~min}$ to $10 \mathrm{~min}$ ) glucose effectiveness (SG), and $\mathrm{S}_{\mathrm{I}}$. Glucose tolerance $(\mathrm{Kg})$ was calculated as the slope of the natural $\log$ of glucose values from min 5 through 19 and this rate expressed as $\% / \mathrm{min}$.

\section{Saliva and urine sampling}

Saliva samples for determination of afternoon and evening free cortisol levels were collected every $30 \mathrm{~min}$ for $8 \mathrm{~h}$, starting $10 \mathrm{~h}$ prior to the subject's scheduled bedtime. Identical (mixed composition) dinners were served just after a saliva 
sample and finished within $40 \mathrm{~min}$, and thus one sample was skipped. Twenty-four hour urine collections were obtained during each visit as well.

\section{Assays}

Serum glucose during the IVGTT was measured using the COBAS Integra 400 (Roche Diagnostics, Indianapolis, IN, USA) with sensitivity of $0.59 \mathrm{mg} / \mathrm{dL}(0.033 \mathrm{mmol} / \mathrm{L})$ and precision $<4.3 \% .{ }^{19}$ Serum insulin was measured by chemiluminescence immunoassay (Access Immunoassay System; Beckman Coulter, Chaska, MN, USA) with sensitivity $0.03 \mathrm{IU} / \mathrm{mL}(0.21$ $\mathrm{pmol} / \mathrm{L}$ ), precision $<5.6 \%$. Salivary cortisol was measured using a solid- phase radioimmunoassay (Coat-A-Count; DPC, Los Angeles, CA, USA), with sensitivity $<0.02 \mu \mathrm{g} / \mathrm{dL}$, and precision $4 \%-5 \%$. The following formula was used to convert to $S_{I}$ units: $\mu \mathrm{g} / \mathrm{dL} \times 27.59=\mathrm{nmol} / \mathrm{L}$. Urinary norepinephrine was assayed using the 2 CAT RIA kit (Immuno Biological Laboratories, Inc, Minneapolis, MN, USA). The sensitivity of this method is $24 \mathrm{pg} / \mathrm{mL}$ for norepinephrine and the precision is $8 \%-15 \%$.

\section{Statistical analysis}

The primary outcome is response to randomized treatment with eszopiclone $(3 \mathrm{mg}$ ) or placebo for 2 months at home, on glucose tolerance, with mechanistic outcomes including IVGTT measures of $S_{\text {I }}$, first phase of insulin secretion, and disposition index. Secondary outcomes, irrespective of treatment, include the relationship of TST and WASO (by PSG, diary, and actigraphy) with glucose metabolism measures.

Two sample $t$-test or Wilcoxon rank sum test (depending on the distribution) was used to compare the change between pre- and post-treatment measures between two treatment groups. Mixed effects models were used to test whether there were significant effects of treatment group and timing (pre vs post). Spearman correlations were calculated to examine the relationships between outcome measures.

\section{Results}

A total of 3121 subjects with a complaint of insomnia were initially screened by telephone; 107 were potentially eligible and were invited for additional screening at Brigham and Women's Hospital. Of these, 53 participants were excluded, as they did not meet the study's inclusion and exclusion criteria after further evaluation (e.g., from sleep diary and actigraphy, laboratory or PSG abnormalities, or diagnosis of comorbid insomnia), and 32 withdrew consent. One subject completed an initial screening visit but did not respond to additional follow-up contacts and another subject withdrew from the study due to the initiation of medical treatment with a non-approved medication.
The study group $(n=20)$ was comprised of nine women and eleven men. The mean age was $39.7 \pm 7.9$ years (range 25-55) and mean BMI was 25.1 2 2.7 (range 20.4-30.1). All subjects had a continuous history of insomnia for at least 6 months, all but one for $>1$ year, and 12/20 for at least 5 years. Their PSQI and ISI scores as well as results of their two overnight sleep studies confirmed their insomnia (Table 1). ${ }^{20}$ Most had no history of a mood or anxiety disorder; however, one subject had a distant history of probable alcohol abuse and another reported a history of depression lasting about 1 year, resolving 10 years prior to the study. A third subject reported having a panic attack in the months prior to the study but was asymptomatic at the time of enrollment.

\section{Medications}

Eight of our 20 subjects with PI had taken at least one dose of a benzodiazepine receptor agonist in their lifetime. None had used any of these medications continuously for longer than 1 month at any time. One subject also had a 2-3 year history of regular treatment with a selective serotonin reuptake inhibitor ending $>5$ years prior to enrollment in the study. All subjects had discontinued these medications for at least 3 months prior to randomization. All female subjects were asked to take and document an approved form of birth control (i.e., condom, oral contraceptive) to prevent the possibility of teratogenic effects of eszopiclone on the fetus.

Table I Demographic and questionnaire data in chronic primary insomnia subjects $(n=20)$

\begin{tabular}{llll}
\hline $\begin{array}{llll}\text { Insomnia patient } \\
\text { characteristics }\end{array}$ & \multicolumn{2}{l}{ Mean (SD) } & p-value \\
\cline { 2 - 3 } & Placebo & Eszopiclone & \\
\hline Gender, female \% & $70 \%$ & $20 \%$ & 0.07 \\
Age, (years) & $39.5(6.7)$ & $39.9(9.3)$ & 0.91 \\
BMI, kg/m & $25.5(2.7)$ & $24.7(2.8)$ & 0.53 \\
Non-Hispanic white, \% & $80 \%$ & $90 \%$ & 1.0 \\
PSQI global & $13.6(2.4)$ & $11.1(3.1)$ & 0.07 \\
PSQI sleep latency & $54.0(31.7)$ & $44.7(26.9)$ & 0.50 \\
PSQI total sleep time, hours & $4.7(0.6)$ & $4.7(1.5)$ & 1.0 \\
Beck Depression Inventory & $6.4(5.2)$ & $6.0(5.1)$ & 0.88 \\
ISI & $16.7(2.6)$ & $16.9(4.5)$ & 0.91 \\
RDI & $2.55(2.54)$ & $4.75(4.74)$ & 0.32 \\
PSG TST & $6.89(0.96)$ & $6.28(0.53)$ & 0.11 \\
PSG WASO & $0.69(0.29)$ & $0.91(0.32)$ & 0.12 \\
Actigraphy TST & $6.59(0.78)$ & $6.59(0.93)$ & 0.99 \\
Actigraphy WASO & $1.18(0.32)$ & $1.18(0.21)$ & 0.99 \\
\hline
\end{tabular}

Notes: 19 participants had evaluable data for the PSQI sleep latency item. All other variables were available in all 20 participants. Participants did not differ significantly (Wilcoxon) on any parameters.

Abbreviations: BMI, body mass index; ISI, Insomnia Severity Index; PI, primary insomnia; PSG, polysomnography; PSQI, Pittsburgh Sleep Quality Inventory; RDI, respiratory disturbance index; SD, standard deviation; TST, total sleep time; WASO, wake after sleep onset. 


\section{Adverse events}

There were no serious adverse events in this study. Of the group who received eszopiclone $(\mathrm{n}=10)$, side effects reported were consistent with those listed on the label information for $3 \mathrm{mg}$ eszopiclone. These included unpleasant aftertaste, headaches, dry mouth, somnolence and dizziness.

\section{Polysomnography}

After randomization, the ten subjects who were randomized to 2-months treatment of $3 \mathrm{mg}$ eszopiclone did not demonstrate significant changes in polysomnographic sleep measures when compared with those randomized to placebo (Table 2).

\section{Diary measures of sleep pre- and post-treatment}

Daily diary measures of self-reported TIB, WASO, number of awakenings, and self-reported TST were calculated for the 3 -week period prior to each inpatient visit 2 months apart so as to be calculated over the same time period as actigraphic assessments. Consistent with the PSG data, there was no difference in sleep parameters by treatment group (Table 3).

\section{Actigraphic measures of sleep in 3 weeks prior to each visit}

The group randomized to 2-months treatment with $3 \mathrm{mg}$ eszopiclone $(\mathrm{n}=10)$ did not demonstrate significant changes

Table 2 Baseline and change in PSG and actigraphy in insomniacs treated with $3 \mathrm{mg}$ eszopiclone versus placebo

\begin{tabular}{|c|c|c|c|c|c|c|c|c|}
\hline \multirow[t]{2}{*}{ Variable } & \multirow[t]{2}{*}{ Unit } & \multicolumn{3}{|l|}{ Eszopiclone } & \multicolumn{3}{|l|}{ Placebo } & \multirow[t]{2}{*}{$p$-value } \\
\hline & & $\begin{array}{l}\text { Pre-Tx }(n=10) \\
\text { mean/SD }\end{array}$ & $\begin{array}{l}\text { Post-Tx }(n=9) \\
\text { mean/SD }\end{array}$ & $\begin{array}{l}\text { Change }(n=9) \\
\text { mean/SD }\end{array}$ & $\begin{array}{l}\text { Pre-Tx } \\
(n=10) \\
\text { mean/SD }\end{array}$ & $\begin{array}{l}\text { Post-Tx } \\
(n=10) \\
\text { mean/SD }\end{array}$ & $\begin{array}{l}\text { Change } \\
(n=10) \\
\text { mean/SD }\end{array}$ & \\
\hline Time in bed & $(\min )$ & $479.9 / 3.0$ & $477.7 / 3.2$ & $-2.8 / 3.5$ & $480.2 / 0.7$ & $478.5 / 2.6$ & $-1.7 / 2.3$ & 0.42 \\
\hline Undefined/artifact & $(\min )$ & II.5/36.2 & $0 / 0$ & $-12.7 / 38.2$ & $0.0 / 0.0$ & $0.0 / 0.0$ & $0.0 / 0.0$ & $0.36^{*}$ \\
\hline Wake & $(\min )$ & $84.9 / 72.9$ & $61.4 / 29.1$ & $-4.0 / 24.0$ & $84.4 / 38.1$ & $89.1 / 28.0$ & $4.7 / 29.2$ & 0.41 \\
\hline NREM I & $(\mathrm{min})$ & $28.1 / 14.9$ & $32.6 / 8.7$ & $2.1 / 11.2$ & $30.4 / 10.1$ & $33.1 / 10.5$ & $2.8 / 7.3$ & $0.97 *$ \\
\hline NREM 2 & (min) & 199.9/52.7 & $228.1 / 44.1$ & $16.1 / 38.0$ & $214.0 / 38.1$ & $205.1 / 33.9$ & $-8.9 / 44.8$ & 0.21 \\
\hline NREM 3 & $(\min )$ & $65.0 / 38.0$ & $61.2 / 34.3$ & $-1.3 / 29.5$ & $69.5 / 25.2$ & $67.4 / 33.0$ & $-1.0 / 37.2$ & 0.97 \\
\hline REM & $(\mathrm{min})$ & $90.4 / 37.8$ & $94.4 / 26.2$ & $-2.3 / 27.5$ & $82.0 / 22.8$ & $83.9 / 25.3$ & $1.9 / 29.3$ & 0.75 \\
\hline PSG total sleep & $(\min )$ & $393.5 / 73.6$ & $416.3 / 29.7$ & $2.9 / 25.5$ & $395.8 / 37.9$ & $389.4 / 27.9$ & $-6.4 / 30.2$ & 0.65 \\
\hline PSG sleep efficiency & $(\%)$ & $81.9 / 15.2$ & $87.1 / 6.1$ & $1.1 / 5.1$ & $82.4 / 7.9$ & $81.4 / 5.8$ & $-1.0 / 6.1$ & 0.42 \\
\hline PSG stage I & (\%) & $7.1 / 3.4$ & $7.8 / 2.5$ & $0.4 / 2.6$ & $7.8 / 3.0$ & $8.6 / 2.9$ & $0.8 / 2.3$ & 0.72 \\
\hline PSG stage 2 & $(\%)$ & $51.9 / 8.7$ & $54.9 / 10.2$ & $1.9 / 5.2$ & $53.6 / 7.6$ & $52.8 / 9.4$ & $-0.9 / 10.1$ & 0.47 \\
\hline PSG stage 3 & (\%) & $18.0 / 11.6$ & $14.5 / 8.2$ & $-1.1 / 6.4$ & $17.6 / 6.0$ & $17.3 / 8.8$ & $-0.3 / 7.7$ & 0.81 \\
\hline PSG stage REM & $(\%)$ & $23.0 / 6.9$ & $22.7 / 6.0$ & $-12 / 5.4$ & $21.0 / 6.7$ & $21.4 / 5.7$ & $0.4 / 8.0$ & 0.63 \\
\hline PSG sleep latency & $(\min )$ & $22.2 / 38.2$ & $9.1 / 12.6$ & $-1.2 / 12.8$ & $15.3 / 15.0$ & $19.6 / 18.2$ & $4.4 / 8.2$ & 0.27 \\
\hline PSG WASO & $(\min )$ & $64.0 / 45.4$ & $52.3 / 33.6$ & $-4.3 / 21.8$ & $69.1 / 38.3$ & $69.5 / 31.6$ & $0.4 / 25.3$ & 0.68 \\
\hline Actigraphy total sleep & $(\min )$ & $354.9 / 60.5$ & $336.0 / 35.2$ & $-18.9 / 52.4$ & $338.5 / 35.2$ & $318.3 / 35.5$ & $-20.2 / 26.6$ & 0.95 \\
\hline Actigraphy WASO & $(\mathrm{min})$ & $78.4 / 21.2$ & $80.2 / 18.3$ & $1.8 / 25.3$ & $98.2 / 38.4$ & 103.8/28.1 & $5.6 / 26.9$ & 0.75 \\
\hline Valid days & (\#) & $11.1 / 6.2$ & 19.3/2.5 & $8.2 / 7.2$ & $15.1 / 6.1$ & 19.9/1.7 & $4.8 / 5.7$ & 0.26 \\
\hline
\end{tabular}

Notes: PSG and actigraphy recordings were collected pre- and post-treatment in subjects randomized to $\sim 2$ months of treatment with 3 mg eszopiclone or placebo. PSG recordings were performed during two inpatient visits occurring immediately pre- and post-treatment. Actigraphy was collected in the 3 weeks prior to and during each visit. $p$-values derived from two-sample $t$-tests or Wilcoxon $(*)$ test for the differences between change in eszopiclone group and change in placebo group.

Abbreviations: NREM, non-REM; PSG, polysomnographic; REM, rapid eye movement; SD, standard deviation; Tx, treatment; WASO, wake after sleep onset.

Table 3 Daily diary of sleep (self-reports)

\begin{tabular}{|c|c|c|c|c|c|c|c|c|c|}
\hline \multirow{2}{*}{$\begin{array}{l}\text { Self-reported } \\
\text { sleep (diary) }\end{array}$} & \multicolumn{4}{|c|}{ Eszopiclone } & \multicolumn{4}{|c|}{ Placebo } & \multirow[t]{2}{*}{$p$-value } \\
\hline & $\mathbf{N}$ & $\begin{array}{l}\text { Pre-Tx } \\
\text { (mean/SD) }\end{array}$ & $\begin{array}{l}\text { Post-Tx } \\
\text { (mean/SD) }\end{array}$ & $\begin{array}{l}\text { Difference } \\
\text { (mean/SD) }\end{array}$ & $\mathbf{N}$ & $\begin{array}{l}\text { Pre-Tx } \\
\text { (mean/SD) }\end{array}$ & $\begin{array}{l}\text { Post-Tx } \\
\text { (mean/SD) }\end{array}$ & $\begin{array}{l}\text { Difference } \\
\text { (mean/SD) }\end{array}$ & \\
\hline TIB (h) & 10 & $7.39 / 0.94$ & $7.42 / 0.51$ & $0.04 / 0.67$ & 10 & $7.55 / 0.83$ & $7.68 / 0.89$ & $0.13 / 0.65$ & 0.54 \\
\hline WASO (min) & 9 & $54.6 / 19.2$ & $39.6 / 25.8$ & $-11.4 / 21.6$ & 10 & $4 I .4 / 17.4$ & $48.0 / 34.2$ & $6.6 / 23.4$ & 0.86 \\
\hline Awakenings (\#) & 9 & $2.12 / 0.91$ & $1.55 / 1.07$ & $-0.54 / 0.78$ & 10 & $2.46 / 1.74$ & $2.34 / 1.53$ & $-0.12 / 2.01$ & 0.28 \\
\hline TST (h) & 9 & $6.28 / 0.53$ & $6.64 / 0.66$ & $0.36 / 0.41$ & 10 & $6.89 / 0.96$ & $6.23 / 0.80$ & $0.09 / 0.55$ & 0.19 \\
\hline Estimated TST (h) & 10 & $6.00 / 0.98$ & $6.45 / 0.77$ & $0.45 / 0.73$ & 10 & $6.23 / 0.80$ & $6.31 / 0.90$ & $0.08 / 0.45$ & 0.90 \\
\hline Daily SD of TST (h) & 9 & $1.07 / 0.52$ & $1.04 / 0.47$ & $-0.03 / 0.50$ & 10 & $0.92 / 0.40$ & $0.93 / 0.36$ & $0.01 / 0.50$ & 0.43 \\
\hline
\end{tabular}

Notes: Daily diary measures of self-reported TIB, WASO, number of awakenings, and estimated sleep duration were calculated for the 3-week period prior to each inpatient visit 2 months apart so as to be over the same time period as actigraphic assessments. One subject in the treatment group had missing diary data. p-values were based on the mixed effects models with group and time as fixed effects and subjects as random effects.

Abbreviations: SD, standard deviation; TIB, time in bed; TST, total sleep time; Tx, treatment; WASO, wake after sleep onset. 
in sleep, as assessed by 3-weeks of wrist actigraphy compared with those randomized to placebo. There were no significant changes over 2 months in TIB (eszopiclone= $-5.2 \pm 48.4 \mathrm{~min} ;$ placebo $=3.1 \pm 41.5 \mathrm{~min}$ ), TST (eszopiclone $=-18.9 \pm 52.4 \mathrm{~min} ;$ placebo $=-20.2 \pm 26.6 \mathrm{~min}), \mathrm{SE}$ (eszopiclone $=-3.8 \pm 7.2 \%$; placebo $=-4.7 \pm 2.4 \%)$, SOL (eszopiclone $=13.0 \pm 17.4 \mathrm{~min}$; placebo $=17.8 \pm 16.8 \mathrm{~min}$ ) or WASO (eszopiclone $=1.8 \pm 25.3 \mathrm{~min}$; placebo $=3.1 \pm 41.5 \mathrm{~min}$ ).

\section{Primary outcomes: insulin secretion and glucose tolerance (IVGTT)}

IVGTT data are presented in Figure $2 \mathrm{Kg}$, the rate of glucose disposal from min 5 to 19 following intravenous glucose injection, at baseline was $2.30 \% \pm 0.34 \% / \mathrm{min}$ in the eszopiclone group versus $1.94 \% \pm 0.06 \% / \mathrm{min}$ in the placebo group. Both mean and pre-post difference data were not normally distributed. Following 2 months of treatment, mean $\mathrm{Kg}$ was $2.63 \% \pm 0.38 \% / \mathrm{min}$ with eszopiclone and $1.84 \% \pm 0.10 \% / \mathrm{min}$ with placebo $(p=0.38$, Wilcoxon two-sample test for group differences and $p=0.31$, Wilcoxon two-sample test for percent change between the two treatment groups). Although the mean difference or percent change is larger in the eszopiclone group, it also has a larger standard deviation. Baseline AIRg was tested as a covariate in the mixed model for sleep diaryreported TIB, WASO, and estimated TST (time slept), but was not significant in any of these models $(p>0.05)$.

Mean changes in $S_{I}$ were $-1.19 \pm 2.57 \pm 0.94$ $(\mathrm{mU} / \mathrm{L})^{-1 *} \mathrm{~min}^{-1}$ with eszopiclone, and $0.05 \pm 3.43$

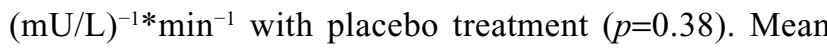
changes in AIRg were $94.0 \pm 269.0 \mathrm{mU}^{*} \mathrm{~L}^{-1 *}$ min with eszopiclone, and $25.1 \pm 74.7 \mathrm{mU}^{*} \mathrm{~L}^{-1 *}$ min with placebo treatment $(p=0.40)$. Mean changes in SG were $0.001 \pm 0.004 \mathrm{~min}^{-1}$ with eszopiclone, and $0.001 \pm 0.009 \mathrm{~min}^{-1}$ with placebo $(p=0.92)$.

Body weight did not change significantly over the course of the 2-month treatment period, or differ between groups. The only significant correlation at baseline was limited to $\mathrm{HbA1c}$ levels and sleep diary-reported estimated TST (time slept), in the placebo group ( $r=-0.66, p=0.0376$ ).

\section{Predictors of changes in $\mathrm{S}_{1}$ over 2 months}

The baseline to post-treatment difference in HbA1c levels was significantly related to the difference in diary-reported estimated TST (time slept) in the eszopiclone-treated group ( $r=0.66, p=0.036)$, and in the combined groups' data $(r=0.55$, $p=0.0125)$. Changes in IVGTT-derived SG were significantly related to the changes in PSG-measured WASO $(r=-0.48$, $p=0.0391$ ) (Figure 3). Other PSG- and actigraphy-derived sleep measures were not correlated with HbA1c or IVGTTderived measures of glucose metabolism.

\section{Salivary cortisol and 24-h urinary epinephrine and norepinephrine levels}

There were no significant effects on salivary cortisol levels of drug versus placebo $(p=0.12)$, baseline versus post-treatment $(p=0.47)$, or drug by time interaction $(p=0.51)$.

There were no significant effects on 24-h urinary epinephrine levels of drug versus placebo $(p=0.53)$, baseline versus post-treatment ( $p=0.49$ ), or drug by time interaction $(p=0.31)$.

There were no significant effects on 24-h urinary norepinephrine levels of drug versus placebo $(p=0.33)$. However, 24-h urinary norepinephrine levels varied with baseline versus post-treatment $(p<0.04)$, and there was a significant drug by time interaction $(p<0.04)$.

\section{Discussion}

In this randomized, double-blind, placebo-controlled and parallel group study of chronic PI patients, nightly administration of $3 \mathrm{mg}$ eszopiclone before bedtime for 2 months did not significantly change indices of glucose metabolism measured using the IVGTT. This may be related to the fact that, in this preliminary study with a small sample size, PSGand diary-derived measures of sleep duration and quality did not differ between eszopiclone and placebo groups. Of note, despite the presence of some mild sleep-disordered breathing in this sample at baseline, this did not differ between groups or alter metabolic responses; eszopiclone has been shown in preliminary work to not alter sleep-disordered breathing severity. ${ }^{20}$ PSG-measured WASO, a hallmark of PI, ${ }^{1}$ was related to IVGTT-derived glucose effectiveness, or non-insulin-mediated glucose uptake, typically by the brain. Other PSG and actigraphic measures of sleep were unrelated to other changes in glucose metabolism. Changes in diaryreported TST were associated with changes in $\mathrm{HbA} 1 \mathrm{c}$ levels, a measure of glycemic control, but were not associated with IVGTT-derived metabolic measures.

Multiple studies have reported an association between sleep restriction in individuals without insomnia, and impaired glucose metabolism, specifically by reductions in $\mathrm{S}_{\mathrm{I}}$ without adequate compensatory increases in insulin secretion. ${ }^{18,21-23}$ It is unclear whether the same mechanisms apply to insomnia. Population-based studies identify a higher risk of diabetes among patients with insomnia, ${ }^{3,24-27}$ though there have been contrary reports from long-term studies. ${ }^{28}$ The direct effects of insomnia on measures of glucose metabolism that might 


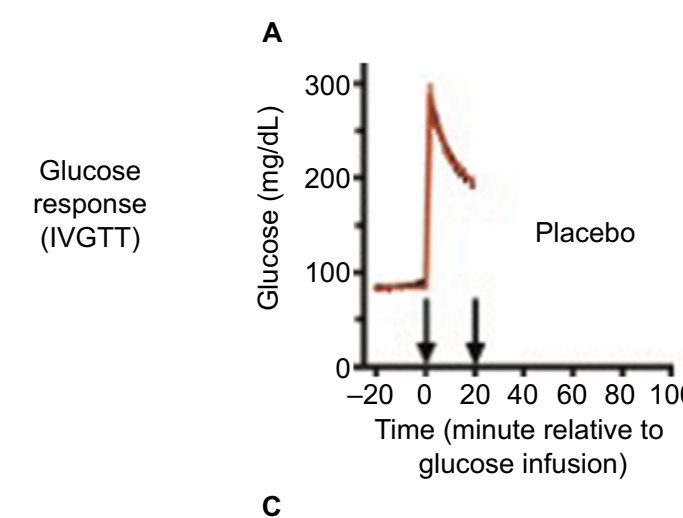

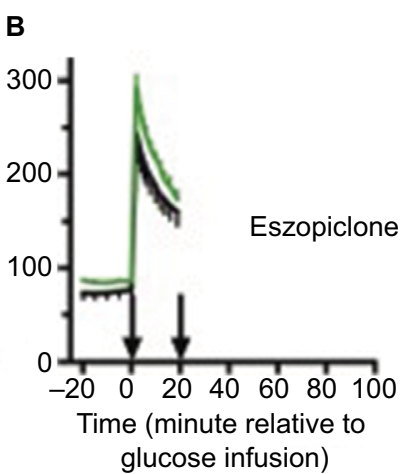

D
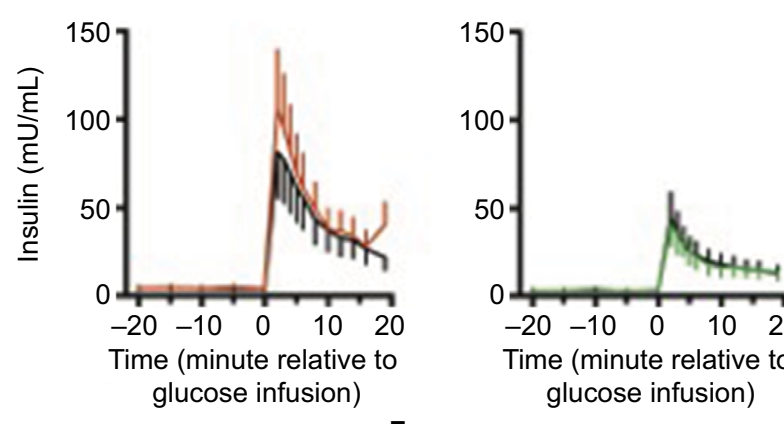

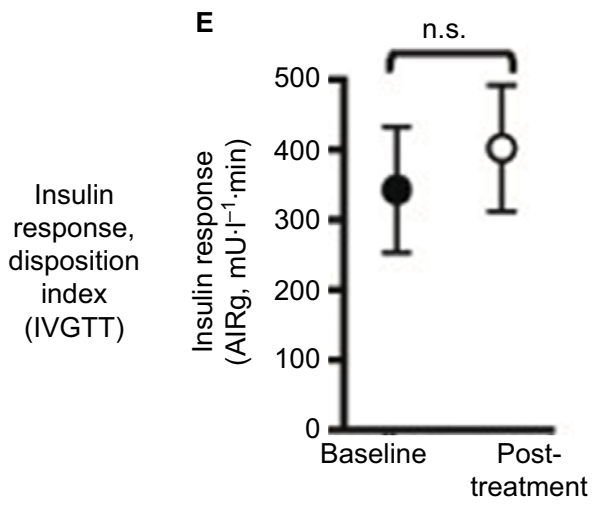

Insulin response, disposition index (IVGTT)

response, first phase (IVGTT)
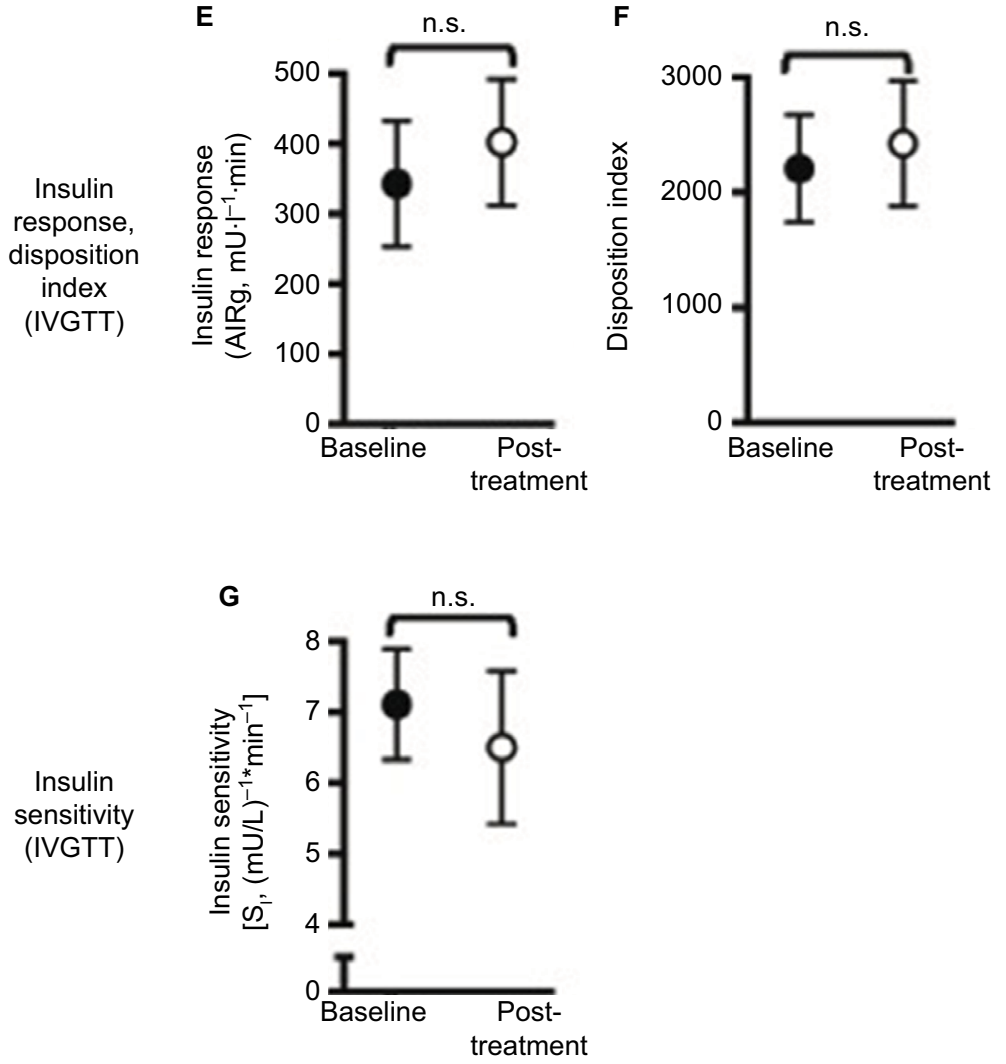

Figure 2 Glucose metabolism in chronic primary insomnia patients $(\mathrm{n}=20)$.

Notes: $(\mathbf{A})$ and (B) Mean glucose levels ( \pm SE) during IVGTT at baseline (black lines) and following 2 months of at-home treatment in chronic primary insomnia patients receiving placebo (A; red line) or eszopiclone (B; green line). Left arrow, glucose infusion at time $t=0$ min; right arrow, insulin infusion at time $t=20$ min. (C) and (D): Mean insulin levels $( \pm S E)$ during the first phase of the insulin response to the IVGTT. (E-G): IVGTT parameters were calculated using the Minmod Millennium 2000 software. (E): AIRg (first phase); (F): Disposition index; (G): Insulin sensitivity. There were no significant effects of drug administration on these metabolic indices.

Abbreviations: AIRg, acute insulin response to glucose; IVGTT, intravenous glucose tolerance test; n.s., not significant; SE, standard error; S, insulin sensitivity.

lead to that elevated diabetes risk are less clear. The combination of short sleep duration $(<6$ h PSG-measured sleep duration during an $8 \mathrm{~h}$ TIB opportunity in the laboratory) and insomnia has been specifically associated with increased diabetes risk. ${ }^{29}$ The results of a recent laboratory study of insomniacs with short sleep and glucose metabolism assessed 

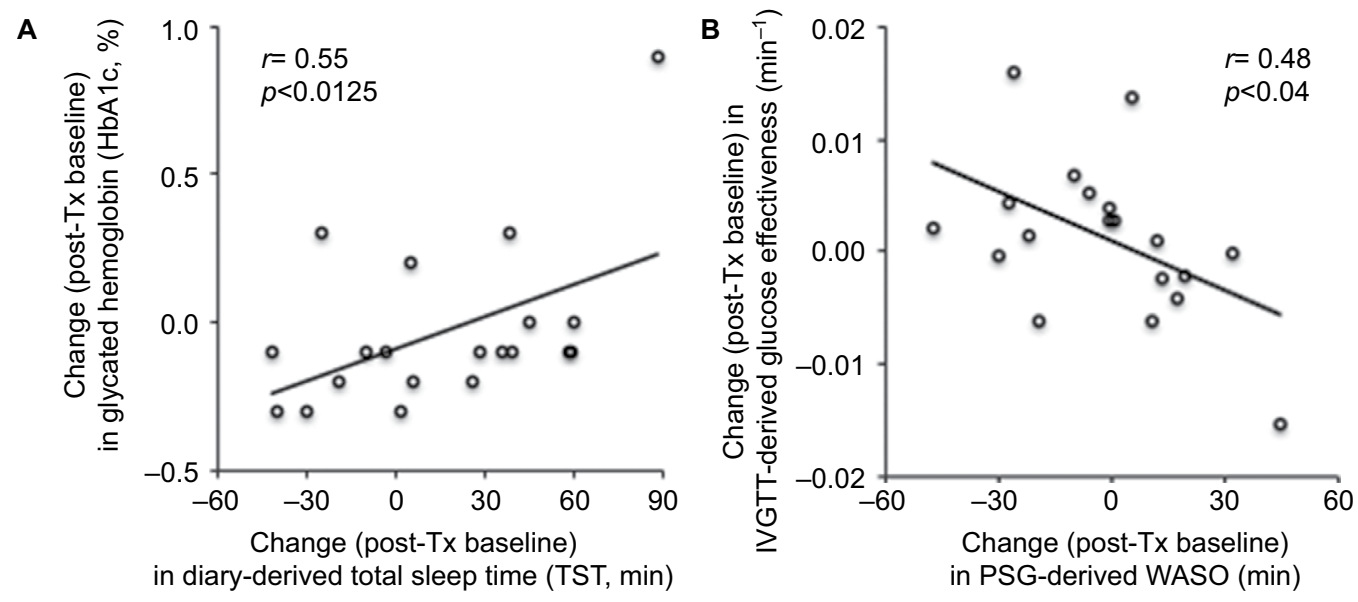

Figure 3 Changes in sleep related to metabolic measures.

Notes: (A): Changes post-Tx in sleep diary-derived TST ( $\mathrm{min}$ ) were significantly correlated with change (post-Tx baseline) in glycated hemoglobin, a measure of glycemic control in which a decrease reflects lower diabetes risk. (B) Changes post-Tx relative to baseline in PSG-derived WASO (min), a hallmark of sleep discontinuity in primary insomnia, were significantly correlated with the IVGTT measure of glucose effectiveness $\left(\mathrm{min}^{-1}\right)$, a measure of non-insulin dependent glucose utilization.

Abbreviations: IVGTT, intravenous glucose tolerance tests; post-Tx, post-treatment; PSG, polysomnography; TST, total sleep time; WASO, wake after sleep onset.

with an oral glucose tolerance test suggest that insomnia with short sleep duration alters glucose metabolism by reducing pancreatic beta cell secretion of insulin, with increased $\mathrm{S}_{\mathrm{I}}{ }^{30}$ This mechanism is inconsistent with the reduction of $S_{I}$ in studies of sleep restriction alone. Interestingly, the reductions in insulin response to a metabolic challenge without changes in $S_{I}$ have also been observed following exposure to circadian disruption, ${ }^{31}$ an exposure that leads to increased sleep fragmentation and shorter sleep duration when sleep occurs at adverse circadian phases. Thus, while sleep restriction and insomnia both elevate diabetes risk, sleep restriction appears to do so via reduced $\mathrm{S}_{\mathrm{I}}$, whereas sleep disruption may elevate diabetes risk by reducing insulin secretion.

Our secondary hypothesis was that improved sleep with eszopiclone would be reflected in improved glucose measures. However, we found no measured effect of eszopiclone on any of the metabolic or sleep measures. Studies that have aimed to assess the clinical efficacy of eszopiclone have included a substantially higher number of patients. For example, a classically cited study on a sustained efficacy of eszopiclone over 6 months had 593 participants taking eszopiclone and 195 taking placebo. ${ }^{32}$ Another study testing efficacy in older individuals randomized 388 participants..$^{33}$ It is also notable that in large population studies, effects have been modest. ${ }^{24,34,35}$ We found that change in measures of sleep quality were a predictor of changes in glycemic control (HbA1c levels) and pancreatic beta cell responses to glucose in our patients with insomnia. Chronic partial sleep loss or insomnia impair glucose metabolism in the short term and are associated with the development of diabetes in the long term. Cross-sectional evidence of the role of short sleep in the development of impaired glucose tolerance and diabetes mellitus type 2 has been demonstrated in the Sleep Heart Health Study. Recently presented oral glucose tolerance test data from this cohort used diagnostic criteria (glucose levels 2-h post-ingestion) for categorizing normal, impaired glucose tolerance, and type 2 diabetes. A self-reported short sleep duration ( $<6 \mathrm{~h}$ night, with or without an insomnia-like symptom), relative to a 7-8 $\mathrm{h}$ sleep duration, was associated with a significant increase in the odds ratio for a worsening of glucose tolerance to impaired glucose tolerance or type 2 diabetes. ${ }^{26}$

Longitudinal evidence of the role of short sleep in the development of type 2 diabetes has been shown in the Nurses Health Study, ${ }^{26}$ as well as a very recent study from Sweden. ${ }^{27}$ In this report, non-diabetic healthy men were followed for a mean period of nearly 15 years. The presence of diabetes was quantified by questionnaire and/or fasting blood glucose levels. Men who reported difficulties falling asleep or regularly used a hypnotic, which was suggestive of sleep problems, were more likely to develop subsequent diabetes even in a model fully adjusted for age, biological risk factors, lifestyle, family history of diabetes, and socioeconomic status. ${ }^{27} \mathrm{DM} 2$ is an epidemic in the US and much of the developed world. ${ }^{36,37}$

It is naturally assumed that patients with chronic insomnia have a shorter overall sleep time. Indeed, they exhibit many of the features that would be expected with a high "sleep debt", including increased alpha power ${ }^{38}$ and relative hypercortisolemia during the afternoon and evening, similar to that seen with acute sleep deprivation ${ }^{39}$ or sleep restriction. ${ }^{18,21}$ The pathophysiological pathway of the changes in glucose control remains to be elucidated. Potential mechanisms include direct effects on the "stress" system, proposed by Basta et al. ${ }^{40} \mathrm{We}$ did 
not find any significant differences in stress hormones: cortisol and norepinephrine remained similar across sleep measures, suggesting that the poorer glycemic control and pancreatic response of insulin secretion is not an effect of HPA activation.

Sleep quality influences the restorative capacity of sleep. ${ }^{41}$ A direct link between sleep quality and diabetes has been shown separately for both difficulty with sleep initiation and difficulty with sleep maintenance. ${ }^{3,25,42-44}$ For example, in an 8-year follow-up of 2265 healthy men, Kawakami et $\mathrm{al}^{42}$ found a more than twice increased risk of type 2 diabetes among individuals who reported difficulty with sleep maintenance and a nearly threefold increased risk among those reporting frequent difficulty with sleep initiation. In the MONICA/KORA Augsburg Cohort Study of a total of 8,269 adults, incident diabetes in multivariable-adjusted models exhibited a hazard ratio of 1.6 (confidence interval [CI]: 1.05-2.45) for men and 1.98 (CI: 1.20-23.29) for women. ${ }^{3}$ In a meta-analysis of sleep-associated diabetes risk, sleep duration exhibited a significant relative risk of 1.28, but insomnia symptoms exhibited a greater degree of diabetes risk. Difficulty initiating sleep showed a relative risk of 1.57, and difficulty maintaining sleep showed a relative risk of $1.84 .^{3}$

Study limitations include incomplete generalizability, as well as the fact that insomnia was defined categorically, and questions the effects of chronicity of insomnia could not be answered. We found no effect of eszopiclone on any of the metabolic or sleep measures that could possibly be attributed to a relatively small number of subjects in each group; that is, our study was underpowered to detect such effects. With ten patients in each group, we have $18 \%$ power to detect the difference in TST of $363.8 \mathrm{~min}(\mathrm{SD}=63.5)$ in placebo group and $411.8 \mathrm{~min}(\mathrm{SD}=124.0)$ versus eszopiclone $3 \mathrm{mg}$ group ${ }^{45}$ with a two-sided two-sample $t$-test at 0.05 level. Similarly, with ten patients in each group, we have $7 \%$ power to detect the difference in WASO of $49.1 \mathrm{~min}(\mathrm{SD}=36.1)$ in placebo group versus $41.2 \mathrm{~min}(\mathrm{SD}=39.0)$ in the eszopiclone $3 \mathrm{mg}$ group ${ }^{45}$ with a two-sided two-sample $t$-test at 0.05 level. Actigraphy was used to detect at-home WASO in the weeks before each inpatient visit, yet this measure, while recently validated, ${ }^{16}$ is weakest for detecting quiet wakefulness during the sleep period and has greater bias (assessed by Bland-Altman plots) with greater amounts of WASO (over 30 min per night), as seen in insomniacs compared with normal sleepers. The strengths of the study include strict and consistent inclusion criteria, sufficiently long treatment and controlled laboratory conditions for comprehensive metabolic testing.

Since poor glucose control can be seen among insomnia patients with shorter sleep times, patients with insomnia who are refractory to treatment should be screened and adequately evaluated for early signs of diabetes; for example, with $\mathrm{HbA1c}$ testing for pre-diabetic levels.

\section{Summary \\ Current knowledge/study rationale}

Insomnia appears to increase diabetes risk. This study tested the hypothesis that the effects of PI on glucose metabolism could be improved by 2 months of pharmacological treatment, and that changes in WASO, a hallmark of insomnia, are related to changes in glucose metabolism.

\section{Study impact}

Preliminary evidence in a relatively small sample presented here suggests that anti-insomnia treatment ( $3 \mathrm{mg}$ eszopiclone every night for 2 months) does not improve glucose metabolism.

\section{Acknowledgments}

We would like to thank the research volunteers for their participation, the Brigham and Women's Hospital General Clinical Research Center and the McLean Hospital Brain Imaging Center technical staff for their assistance with data collection. We further acknowledge the contributions of the late Emily Reid.

This work was supported by the Frank Gillis Fund, the Florence Petrlik Charitable Foundation, an investigatorinitiated research grant from Sunovion Pharmaceuticals Inc. (formerly Sepracor) and was conducted in the General Clinical Research Center supported by the National Center for Research Resource (NCRR M01 RR02635). The content is solely the responsibility of the authors and does not necessarily represent the official views of the NCRR or National Institutes of Health.

\section{Disclosure}

Although this investigator-initiated study was sponsored in part by Sunovion Pharmaceuticals Inc. (formerly Sepracor), the authors analyzed the data and wrote the paper.

OMB has received two investigator-initiated grants from Sepracor Inc. (now Sunovion; ESRC-0004 and ESRC-0977, ClinicalTrials.gov Identifiers NCT00555750, NCT00900159), and two investigator-initiated grants from Cephalon Inc. (now Teva; ClinicalTrials.gov Identifier: NCT00895570). OMB received Speaker's Bureau, CME and non-CME lecture honoraria and an unrestricted educational grant from Takeda Pharmaceuticals, North America. OMB served as a consultant and expert witness for Dinsmore LLC, and received consulting fees for serving on the Scientific Advisory Board of Matsutani America and from the Wake Forest University Medical Center (NC). OMB received speaking fees and/or travel support for 
speaking from Chevron; American Academy of Craniofacial Pain; National Heart, Lung, and Blood Institute; National Institute of Diabetes and Digestive and Kidney Diseases; National Postdoctoral Association; Oklahoma State University; Oregon Health Sciences University; SUNY Downstate Medical Center; American Diabetes Association; and New York University.

JWW has participated in research sponsored by Glaxo SmithKline, UCB Pharma, and Impax Pharmaceuticals. He has been a consultant for Impax Pharmaceuticals and UCB Pharma, and part of the scientific advisory board for Zeo Inc. The other authors report no conflict of interests in this work.

\section{References}

1. Lyytikainen P, Lallukka T, Lahelma E, Rahkonen O. Sleep problems and major weight gain: a follow-up study. Int J Obes (Lond). 2011; 35(1):109-114.

2. Ayas NT, White DP, Al-Delaimy WK, et al. A prospective study of self-reported sleep duration and incident diabetes in women. Diabetes Care. 2003;26(2):380-384.

3. Cappuccio FP, D'Elia L, Strazzullo P, Miller MA. Quantity and quality of sleep and incidence of type 2 diabetes: a systematic review and meta-analysis. Diabetes Care. 2010;33(2):414-420.

4. Anothaisintawee T, Reutrakul S, Van Cauter E, Thakkinstian A. Sleep disturbances compared to traditional risk factors for diabetes development: systematic review and meta-analysis. Sleep Med Rev. 2015; 30:11-24.

5. Senthilvel E, Auckley D, Dasarathy J. Evaluation of sleep disorders in the primary care setting: history taking compared to questionnaires. $J$ Clin Sleep Med. 2011;7(1):41-48.

6. Spielman AJ, Caruso LS, Glovinsky PB. A behavioral perspective on insomnia treatment. Psychiatr Clin North Am. 1987;10(4):541-553.

7. Riemann D, Spiegelhalder K, Feige B, et al. The hyperarousal model of insomnia: a review of the concept and its evidence. Sleep Med Rev. 2010; 14(1):19-31.

8. Pavlova M, Berg O, Gleason R, Walker F, Roberts S, Regestein Q. Selfreported hyperarousal traits among insomnia patients. J Psychosom Res. 2001;51(2):435-441

9. Hantsoo L, Khou CS, White CN, Ong JC. Gender and cognitive-emotional factors as predictors of pre-sleep arousal and trait hyperarousal in insomnia. J Psychosom Res. 2013;74(4):283-289.

10. Killgore WD, Schwab ZJ, Kipman M, Deldonno SR, Weber M. Insomnia-related complaints correlate with functional connectivity between sensory-motor regions. Neuroreport. 2013;24(5):233-240.

11. Spiegelhalder K, Regen W, Feige B, et al. Increased EEG sigma and beta power during NREM sleep in primary insomnia. Biol Psychol. 2012;91(3):329-333.

12. Buysse DJ, Nofzinger EA, Germain A, et al. Regional brain glucose metabolism during morning and evening wakefulness in humans: preliminary findings. Sleep. 2004;27(7):1245-1254.

13. Vgontzas AN, Tsigos C, Bixler EO, et al. Chronic insomnia and activity of the stress system: a preliminary study. J Psychosom Res. 1998; 45(1):21-31.

14. International Expert Committee. International Expert Committee report on the role of the A1Cassay in the diagnosis of diabetes. Diabetes Care. 2009;32(7):1327-1334.

15. Bergman RN. Pathogenesis and prediction of diabetes mellitus: lessons from integrative physiology. Mt Sinai J Med. 2002;69(5):280-290.

16. Marino MM, Li Y, Rueschman MN, et al. Measuring sleep: accuracy, sensitivity, and specificity of wrist actigraphy compared to polysomnography. Sleep. 2013;36(11):1747-1755.

17. St Hilaire MA, Klerman EB. Inter-individual variability in the parameters of a mathematical model of neurobehavioral performance and alertness. Sleep. 2007;30(abstr Suppl):A52.
18. Buxton OM, Pavlova M, Reid EW, Wang W, Simonson DC, Adler GK. Sleep restriction for 1 week reduces insulin sensitivity in healthy men. Diabetes. 2010;59(9):2126-2133.

19. Caraway WT, Watts NB. Carbohydrates. In: Tietz NW, editor. Fundamentals of Clinical Chemistry. 3rd ed. Philadelphia: WB Saunders; 1987:422-447.

20. Rosenberg R, Roach JM, Scharf M, Amato DA. A pilot study evaluating acute use of eszopiclone in patients with mild to moderate obstructive sleep apnea syndrome. Sleep Med. 2007;8(5):464-470.

21. Spiegel K, Leproult R, Van Cauter E. Impact of sleep debt on metabolic and endocrine function. Lancet. 1999;354(9188):1435-1439.

22. Nedeltcheva AV, Kessler L, Imperial J, Penev PD. Exposure to recurrent sleep restriction in the setting of high caloric intake and physical inactivity results in increased insulin resistance and reduced glucose tolerance. J Clin Endocrinol Metab. 2009;94(9):3242-3250.

23. Buxton OM, Broussard JL, Zahl AK, Hall M. Effects of sleep deficiency on hormones, cytokines, and metabolism. In: Redline S, Berger NA, editors. Energy Balance and Cancer Volume 8: Impact of Sleep and Sleep Disturbances on Obesity and Cancer. New York, NY: Springer; 2013:25-50.

24. Vgontzas AN, Liao D, Pejovic S, Calhoun S, Karataraki M, Bixler EO. Insomnia with objective short sleep duration is associated with type 2 diabetes: apopulation-based study. Diabetes Care. 2009;32(11):1980-1985.

25. Meisinger C, Heier M, Loewel H. Sleep disturbance as a predictor of type 2 diabetes mellitus in men and women from the general population. Diabetologia. 2005;48(2):235-241.

26. Gottlieb DJ, Punjabi NM, Newman AB, et al. Association of sleep time with diabetes mellitus and impaired glucose tolerance. Arch Intern Med. 2005;165(8):863-867.

27. Nilsson PM, Roost M, Engstrom G, Hedblad B, Berglund G. Incidence of diabetes in middle-aged men is related to sleep disturbances. Diabetes Care. 2004;27(10):2464-2469.

28. Björkelund C, Bondyr-Carlsson D, Lapidus L, et al. Sleep disturbances in midlife unrelated to 32-year diabetes incidence: the prospective population study of women in Gothenburg. Diabetes Care. 2005;28(11):2739-2744.

29. Dang-Vu TT, McKinney SM, Buxton OM, Solet JM, Ellenbogen JM. Spontaneous brain rhythms predict sleep stability in the face of noise. Curr Biol. 2010;20(15):R626-R627.

30. Vasisht KP, Kessler LE, Booth JN 3rd, Imperial JG, Penev PD. Differences in insulin secretion and sensitivity in short-sleep insomnia. Sleep. 2013;36(6):955-957.

31. Buxton OM, Cain SW, O'Connor SP, et al. Adverse metabolic consequences in humans of prolonged sleep restriction combined with circadian disruption. Sci Transl Med. 2012;4(129):129ra43.

32. Krystal AD, Walsh JK, Laska E, et al. Sustained efficacy of eszopiclone over 6months of nightly treatment: results of a randomized, doubleblind, placebo- controlled study in adults with chronic insomnia. Sleep. 2003;26(7):793-799.

33. Ancoli-Israel S, Krystal AD, McCall WV, et al. A 12-week, randomized, double- blind, placebo-controlled study evaluating the effect of eszopiclone $2 \mathrm{mg}$ on sleep/wake function in older adults with primary and comorbid insomnia. Sleep. 2010;33(2):225-234.

34. Vgontzas AN, Fernandez-Mendoza J, Liao D, Bixler EO. Insomnia with objective short sleep duration: the most biologically severe phenotype of the disorder. Sleep Med Rev. 2013;17(4):241-254.

35. Knutson KL, Van Cauter E, Zee P, Liu K, Lauderdale DS. Cross-sectional associations between measures of sleep and markers of glucose metabolism among subjects with and without diabetes. Diabetes Care. 2011; 34:1171-1176.

36. King H, Zimmet P. Trends in the prevalence and incidence of diabetes: non- insulin-dependent diabetes mellitus. World Health StatQ. 1988; 41(3-4):190-196.

37. King H, Aubert RE, Herman WH. Global burden of diabetes, 1995-2025:prevalence, numerical estimates, and projections. Diabetes Care. 1998;21(9):1414-1431.

38. Aeschbach D, Sher L, Postolache TT, Matthews JR, Jackson MA, Wehr TA. A longer biological night in long sleepers than in short sleepers. J Clin Endocrinol Metab. 2003;88(1):26-30. 
39. Leproult R, Copinschi G, Buxton O, Van Cauter E. Sleep loss results in an elevation of cortisol levels the next evening. Sleep. 1997;20(10):865-870.

40. Basta M, Chrousos GP, Vela-Bueno A, Vgontzas AN. Chronic insomnia and stress system. Sleep Med Clin. 2007;2(2):279-291.

41. Akerstedt T, Nilsson PM. Sleep as restitution: an introduction. J Intern Med. 2003;254(1):6-12.

42. Kawakami N, Takatsuka N, Shimizu H. Sleep disturbance and onset of type 2 diabetes. Diabetes Care. 2004;27(1):282-283.

43. Mallon L, Broman JE, Hetta J. High incidence of diabetes in men with sleep complaints or short sleep duration: a 12-year follow-up study of a middle-aged population. Diabetes Care. 2005;28(11):2762-2767.
44. Hayashino Y, Fukuhara S, Suzukamo Y, Okamura T, Tanaka T, Ueshima H. Relation between sleep quality and quantity, quality of life, and risk of developing diabetes in healthy workers in Japan: the High-risk and Population Strategy for Occupational Health Promotion (HIPOP-OHP) Study. BMC Public Health. 2007;7:129.

45. Zammit GK, McNabb LJ, Caron J, Amato DA, Roth T. Efficacy and safety of eszopiclone across 6-weeks of treatment for primary insomnia. Curr Med Res Opin. 2004;20(12):1979-1991.

\section{Publish your work in this journal}

Nature and Science of Sleep is an international, peer-reviewed, open access journal covering all aspects of sleep science and sleep medicine, including the neurophysiology and functions of sleep, the genetics of sleep, sleep and society, biological rhythms, dreaming, sleep disorders and therapy, and strategies to optimize healthy sleep. The manuscript

\section{Dovepress}

management system is completely online and includes a very quick and fair peer-review system, which is all easy to use. Visit http://www. dovepress.com/testimonials.php to read real quotes from published authors. 